\title{
A CASE REPORT: ICTAL BRADYCARDIA IN A PATIENT WITH SYNCOPAL ATTACKS
}

\author{
Koray Demirci¹, Babürhan Güldiken²
}

${ }^{1}$ Trakya University Faculty of Medicine, Edirne, TURKEY

${ }^{2}$ Department of Neurology, Trakya University Faculty of Medicine, Edirne, TURKEY

\section{ABSTRACT}

Aims: Ictal bradycardia is characterized as the presence of bradycardia during the ictal discharge and mostly seen in left temporal zone originated seizures. It could lead to conditions such as syncope, asystole and severe injuries. With this case report, it is aimed to emphasize the importance of applying 24-hour video electroencephalographyelectrocardiography monitoring in patients with unexplained loss of consciousness.

Case Report: A 68-year old male patient was admitted to Trakya University Hospital with a history of falls and loss of consciousness for one year. The patient underwent six days long 24-hour video- electroencephalographyelectrocardiography monitoring and on the second day, an ictal discharge on left temporal zone accompanied by a heart beat decrease was seen.

Conclusion: This case shows the importance of 24-hour video-electroencephalography-electrocardiography monitoring in diagnosis of ictal bradycardia. Treating these patients with antiepileptic drugs could prevent the severe complications of the condition. In case of insufficiency of the antiepileptic drug therapy, inserting a cardiac pacemaker may be considered.

Keywords: Seizures, bradycardia, aphasia

\section{INTRODUCTION}

Ictal epileptic discharges could cause cardiac arrhythmias especially in patients whose seizures are originated in temporal zone (1). It has been reported that $64-100 \%$ of the temporal lobe seizures are presented with ictal tachycardia $(1,2)$. On the other hand, ictal bradycardia is occasionally seen mostly as a component of complex partial seizures originated in temporal or frontal zones and accounts for $<2 \%$ of the epileptic patients (3).

Ictal bradycardia is a serious complication, as it could lead to cardiac asystole, syncope and is also accused for Sudden Unexpected Death in Epilepsy (SUDEP) (1-5). Diagnosing ictal bradycardia requires electroencephalography (EEG) - electrocardiography (ECG) monitoring to detect bradycardia during the ictal discharge. Nevertheless, because of EEG-ECG monitoring is not routinely done and the course of ictal bradycardia could be asymptomatic, misdiagnosis is common $(1,5)$. Furthermore, due to the rarity of reports regarding ic- tal bradycardia cases, there are no guidelines about the management of the disease (2). To prevent the course to syncope and falls, avoiding misdiagnosing and applying appropriate management to patients are crucial.

Hereby, we report a case who has a previous history of seizures with syncope and therefore, is hospitalized to undergo 24-hour video-EEG monitoring which revealed ictal bradycardia following a left temporal lobe ictal discharge.

\section{CASE REPORT}

A 68-year-old male patient was admitted to Trakya University Hospital with a history of episodes characterized by nausea, fatigue and aphasia which lasted approximately one minute. The patient began having episodes one year ago, which occurred 5-6 times during the first month after his first seizure. During the subsequent 6 months, the patient experienced no attack. However, 
4 months ago, he had a fall presented with loss of consciousness, nausea and urinary incontinence with a duration of time less than 10 minutes. The patient had no previous history of cardiovascular diseases or diabetes, but had a meningitis infection in his childhood. His mother died due to a cerebrovascular disease, while his two brothers have lung cancer.

Neurological examination was unremarkable. The patient underwent 24-hour video-EEG monitoring for six days. His interictal EEG revealed paroxysmal 2-3 Hz temporal sharp-slow waves on a normal background activity (Figure 1A). On the second day of monitoring, a 38 second lasting seizure with an abdominal aura was recorded, while the patient was lying supine in an awake state. The ictal activity began on the left temporal area 16 seconds later after the aura (Figure 1B), and five seconds after the ictal discharge, the heart beat decreased from 76 beats per minute to 52 beats per minute and the ictal bradycardia persisted 20 seconds (Figure 1C). After the end of the ictal EEG discharge, the heart rate returned to normal rate. The patient reported that he could hear the voices during the seizure, but could not speak although he wanted to (Figure 2). He also informed that his previous seizures began with the same complaints, but persisted longer and ended in loss of consciousness. No seizure was observed in the other days of his stay. There were no pathological findings on cranial MRI report.

As the differential diagnosis of the patient, ictal bradycardia, ictal aphasia and subsequent ictal syncope were considered. In respect of the initial therapy, levatirasetam was given to the patient, however discontinued when patient stated that he could not tolerate the medicine and had ataxia while walking. Instead of levatirasetam, lamotrigine was given to the patient. For the six months follow up, no seizures and no syncopal attacks have been reported.

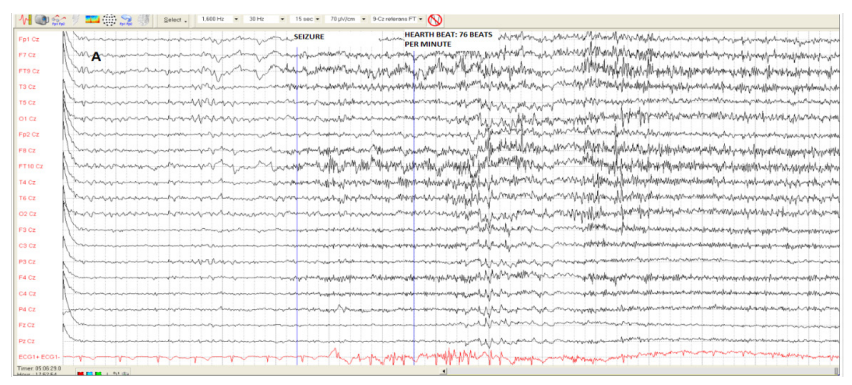

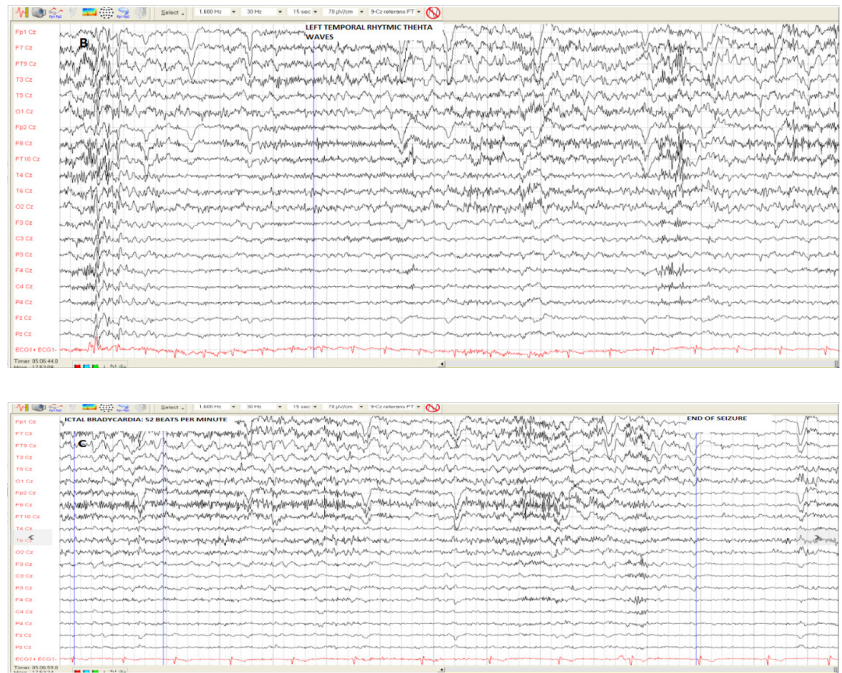

Figure 1A,B,C: The EEG-ECG findings of the patient, when patient had seizure on the second day of monitoring

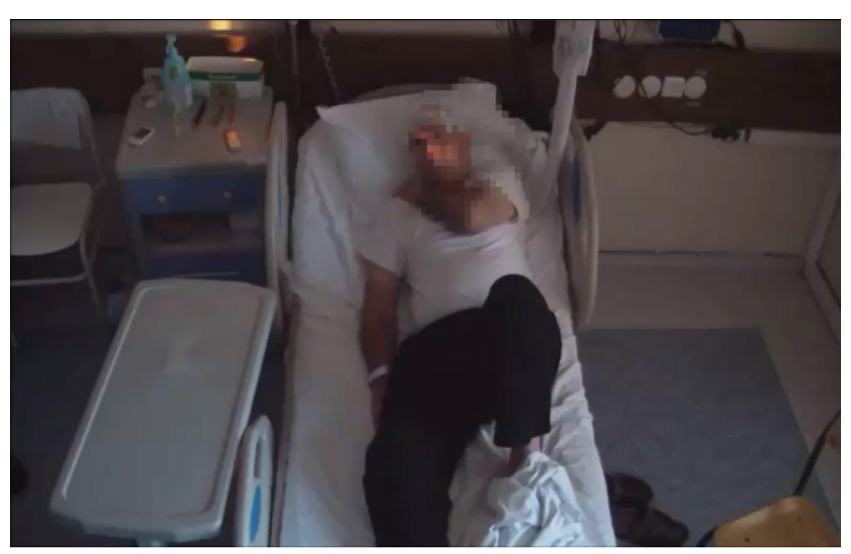

Figure 2: The video record of the patient when having seizure with ictal bradycardia (You can watch the video either by clicking the link on the figure or opening the page: https://youtu.be/p4cL5wl7k68)

\section{DISCUSSION}

Although ictal bradycardia does not seem to be a particular concern, it could lead to life-threatening conditions such as syncope, asystole. Therefore is accused in etiology of SUDEP (1-5). Diagnosing ictal bradycardia remains challenging, as EEG-ECG simultaneous monitoring is not a routine in most centers. In elderly, episodes of loss of consciousness may usually occur due to epileptic seizure or syncope. Clinical manifestations 
such as falls, convulsions, urinary incontinance are not entirely specific for an entity, and cannot be used reliably in differential diagnosis. Unexplained cases should be an aspect to suspect ictal bradycardia, since it is one of the rare causes of decreased heart rate. Our patient reported that he had seizures for the last one year, which presented with falls sometimes. Therefore applying 24-hour video-EEG-ECG monitoring is suggested and out of total six days on the second day ictal bradycardia was detected. Reporting our case, we aimed to emphasize that long term 24-hour video EEG-ECG monitoring plays a critical role in diagnosing ictal bradycardia and is important to prevent life-threatening conditions such as asystole and falls.

Ictal bradycardia occurs mostly in patients, whose seizures are originated in left temporal lobe $(1,5)$. In the review and case report of Tinuper et al. (1) out of 32 cases 20 of them had discharges based on left side. In this aspect, our case is consistent with the literature, as bradycardia occurred when rhythmic theta wave activity in the left temporal area became apparent.

Due to the rarity of the condition, there are no guidelines established in treatment (2). However, in literature it is reported that threatening patients with an antiepileptic drug and a cardiac pacemaker could prevent the life-threating conditions $(1,2,4,5)$. Nevertheless, some antiepileptic drugs could be avoided due to their worsening effect on bradycardia. Carbamezapine could lengthen Q-T interval in ECG, therefore could increase the arrhythmic effects and rise the risk of $\operatorname{SUDEP}(1,3)$. Along with carbamezapine, phenytoin is also not suggested, as it decreases the hyperactivity of the cardiac sympathetic nerves and could worsen the situation (1, 3 ). We administered levasiteram to our patient firstly; but after he stated that he could not tolerate the drug and had ataxia while walking, we changed the drug to lamotrigine. We followed-up the patient six months and observed no seizures and syncopal attacks. Thus, we did not consider placing a cardiac pacemaker as a necessity in our patient.

As a conclusion, it could be stated applying 24-hour video EEG-ECG plays an important role in diagnosing ictal bradycardia and preventing life-threatening conditions like syncope and asystole. The patient should be given an antiepileptic drug followed-up for the effectiveness of the therapy, especially on cardiac symptoms. In case of failure of the antiepileptic drug, inserting a cardiac pacemaker to the patient may be necessary.
Ethics Committee Approval: N/A

Informed Consent: Written informed consent was obtained from the participant of this study.

Conflict of Interest: The authors declared no conflict of interest.

Financial Disclosure: The authors declared that this study received no financial support.

\section{REFERENCES}

1. Tinuper P, Bisulli F, Cerullo A et al. Ictal bradycardia in partial epileptic seizures: autonomic investigation in three cases and a literature review. Brain 2001;124:236171.

2. Fava I, Gaudio SD, Volpe E et al. The ictal bradycardia syndrome: a case report. Epilepsy \& Behaviour 2015;4:912.

3. Devinsky O. Effects of seizures on autonomic and cardiovascular function. Epilepsy Curr 2004;4(2):43-6.

4. Mondon K, Charbonnier B, Hommet C et al. Ictal bradycardia followed by cardiac asystole: a case report. Epileptic Disord 2002;4(4):261-4.

5. Almansori M, Ijaz M, Ahmed SN et al. Cerebral arrhythmia influencing cardiac rhythm: a case of ictal bradycardia. Seizure 2006;15:459-61. 